\title{
Das Europäische Parlament in der öffentlichen Meinung - bekannt aber wenig relevant
}

\author{
Oskar Niedermayer*
}

Das Europäische Parlament hat im Rahmen des EU-Verflechtungssystems drei zentrale Aufgaben: die Politikgestaltung, die Systemgestaltung und die Interaktion mit den Bürgerinnen und Bürgern der Europäischen Union. ${ }^{1}$ Die Interaktionsfunktion umfasst zwei Komponenten: zum einen die Artikulation und Aggregation von Bürgerinteressen und zum anderen die Vermittlung von politischen Entscheidungen an die Bürger. Ausfluss der Erfüllung dieser Funktion auf Seiten der Bevölkerung sind bestimmte Orientierungen kognitiver und evaluativer Art, die die Bürgerinnen und Bürger gegenüber dem Europäischen Parlament entwickeln. Diese Orientierungen sollen im Folgenden untersucht werden, wobei das Hauptaugenmerk auf längerfristigen Entwicklungen liegt und die Orientierungen der Deutschen in der Regel auch in den gesamteuropäischen Rahmen gestellt werden sollen.

Hauptdatenquelle für die hier präsentierten empirischen Ergebnisse sind die seit dem Herbst 1973 halbjährlich im Auftrag der Europäischen Kommission in allen Mitgliedstaaten der Europäischen Union durchgeführten repräsentativen Bevölkerungsumfragen (,Eurobarometer"). ${ }^{2}$ Daneben werden die von der Forschungsgruppe Wahlen e.V. seit der ersten Direktwahl des Europäischen Parlaments durchgeführten Vorwahlumfragen berücksichtigt. ${ }^{3}$

In einem ersten Schritt werden die kognitiven Orientierungen analysiert, also die Bekanntheit des Europäischen Parlaments, das Wissen über diese Institution und die Perzeption seiner Rolle im EU-Institutionensystem. In einem zweiten Schritt folgen die evaluativen Orientierungen, also die positiven oder negativen Bewertungen des Europäischen Parlaments. Dabei stehen die affektiv-wertbezogenen Beurteilungen am Beispiel des Vertrauens in das Europäische Parlament im Mittelpunkt, da für die zweite Art der evaluativen Orientierungen, die eher rational-ergebnisbezogenen Beurteilungen, zu wenig über einen längeren Zeitraum erhobene Daten existieren.

\section{Kognitive Orientierungen gegenüber dem Europäischen Parlament}

\section{Bekanntheitsgrad}

Voraussetzung für jede andere Art von Orientierungen gegenüber einem Objekt ist zunächst die psychologische Hinwendung im Sinne seiner bloßen Wahrnehmung. Seit 1977 werden die Bürgerinnen und Bürger Europas gefragt: „Können Sie sich erinnern, in letzter Zeit in Zeitungen, Zeitschriften, Radio oder Fernsehen irgendetwas über das Europäische

* Prof. Dr. Oskar Niedermayer, Professor für Politische Wissenschaft mit dem Schwerpunkt Politische Soziologie, Freie Universität Berlin.

1 Vgl. schon Eberhard Grabitz/Otto Schmuck/Sabine Steppat/Wolfgang Wessels: Direktwahl und Demokratisierung. Eine Funktionenbilanz des Europäischen Parlaments nach der ersten Wahlperiode, Bonn 1988. Zur Interaktion mit der Bevölkerung als parlamentarischer Schlüsselfunktion vgl. auch Georg Kofler: Das Europäische Parlament und die öffentliche Meinung, Wien/Köln/Graz 1983.

2 Die Umfrageergebnisse werden in Berichten der Kommission veröffentlicht. In jedem Land werden etwa 1.000 Personen ab 15 Jahren befragt (in Deutschland 1.500, in Luxemburg, Malta und Zypern 500 und im Vereinigten Königreich 1.300).

3 Vgl. die Berichte zu den jeweiligen Wahlen, z.B. für 2009: Forschungsgruppe Wahlen e.V. (Hrsg.): Europawahl. Eine Analyse der Wahl vom 7. Juni 2009, Mannheim 2009. 
Parlament gelesen oder gehört zu haben?" Als Wahrnehmungsindikator ist diese Art der Frageformulierung allerdings nicht optimal, da sie auf die kurzfristige, subjektiv erinnerte massenmediale Vermittlung von Informationen über das Europäische Parlament abstellt. Veränderungen der Antwortmuster im Zeitablauf können daher sowohl auf Veränderungen der Intensität des Informationsangebots der Medien als auch auf Veränderungen des Grades an Sensibilisierung der Befragten gegenüber diesem Angebot basieren.

Das Schaubild 1 zeigt die Entwicklung bis 2002. Auch wenn die Datenbasis bis Anfang der Achtzigerjahre sehr unvollständig ist, zeigen die Veränderungen dieses Indikators sowohl für Deutschland als auch für den gewichteten EU-Durchschnitt deutlich die jeweiligen Wahrnehmungsschübe im Umfeld der verschiedenen Direktwahlen. Dem jeweiligen Anstieg der EP-Wahrnehmung anlässlich der Wahlen folgte jedoch immer ein deutlicher Abfall nach den Wahlen, das heißt es war bis 2002 noch nicht gelungen, die kurzfristige Steigerung der Informationsvermittlung und/oder der Sensibilisierung der Bürger gegenüber diesem Informationsangebot in eine längerfristig stabile Wahrnehmung des Europäischen Parlaments auf einem höheren Niveau zu transformieren. Bedenklich stimmt zudem, dass der Wahrnehmungsschub ab der dritten Direktwahl weitaus geringer ausfiel als bei den ersten beiden Wahlen.

\section{Schaubild 1: Wahrnehmung des Europäischen Parlaments 1977-2002 (Angaben in Prozent)}

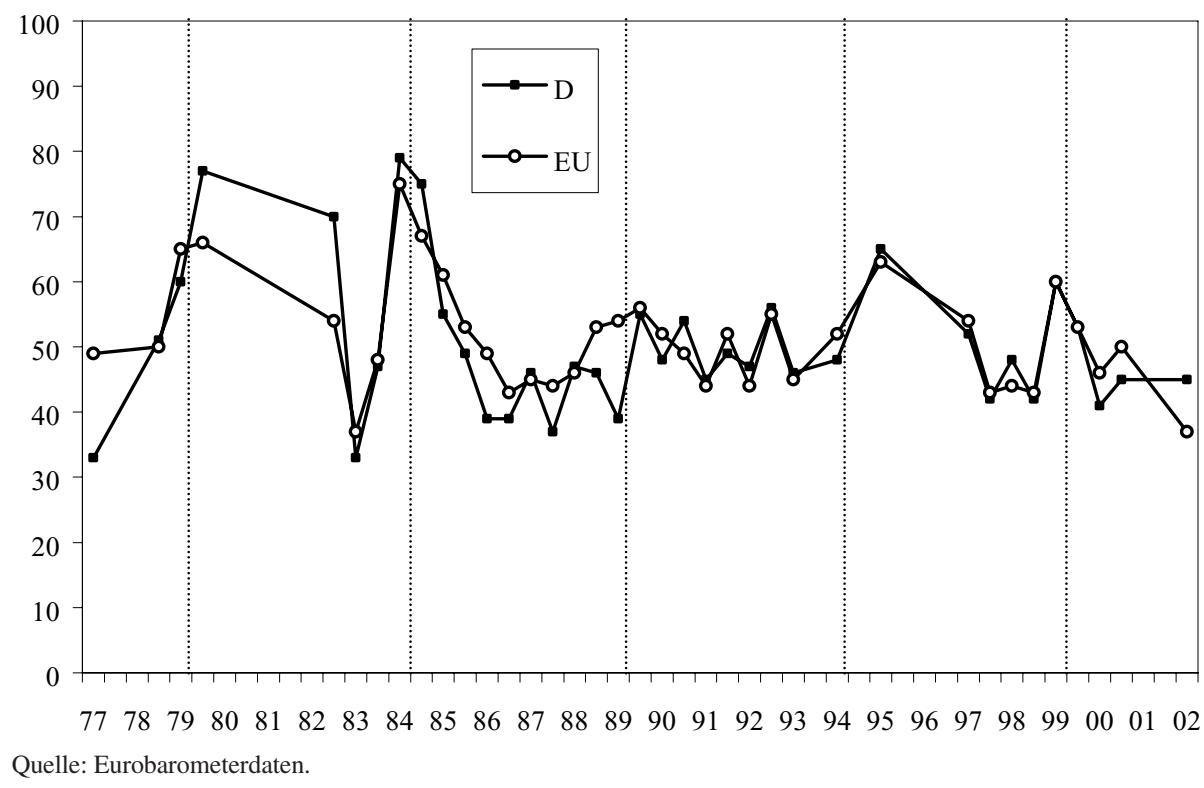

Nachdem diese Frage längere Zeit nicht gestellt worden war, ist sie im Eurobarometer 70 vom Herbst 2008 wieder enthalten. ${ }^{4}$ Die Ergebnisse - 50 Prozent der Deutschen und 44 Prozent im EU-Durchschnitt können sich erinnern, in letzter Zeit in den Medien etwas über das

4 Europäische Kommission: Eurobarometer 70. Die öffentliche Meinung in der Europäischen Union. Erste Ergebnisse, Dezember 2008; Europäische Kommission: Table of Results. First Results: Standard Eurobarometer 70, Dezember 2008. 
Europäische Parlament gelesen oder gehört zu haben - zeigen, dass sich am Niveau der Wahrnehmung bis heute nichts geändert hat.

Seit 1999 wird der Bekanntheitsgrad des Europäischen Parlaments über die simple Frage „Haben Sie schon einmal etwas über das Europäische Parlament gehört oder gelesen?“ gemessen. Damit wird im Gegensatz zur ersten Frage vor allem keinerlei Zeitbezug gesetzt und zudem wird weniger explizit auf die massenmediale Berichterstattung abgestellt, also auch mögliche interpersonale Kommunikation über das Europäische Parlament einbezogen. Damit werden die Anforderungen an eine positive Antwort insgesamt möglichst gering gehalten. Schaubild 2 zeigt daher auch, dass sowohl in Deutschland als auch in der ganzen Europäischen Union etwa neun von zehn Bürgern vom Europäischen Parlament gehört haben. Bei diesem hohen Bekanntheitsgrad können Ereignisse wie die Direktwahlen nur noch einen begrenzten Effekt haben. Nach der Direktwahl von 2004 stieg der Bekanntheitsgrad bei den Deutschen allerdings knapp über den europäischen Durchschnitt und blieb seither auch dort, wobei die EU-Osterweiterung am 1. Mai 2004 sich auf den EU-Durchschnitt nicht unmittelbar auswirkte.

\section{Schaubild 2: Bekanntheitsgrad des Europäischen Parlaments 1999-2008 (Angaben in Prozent)}

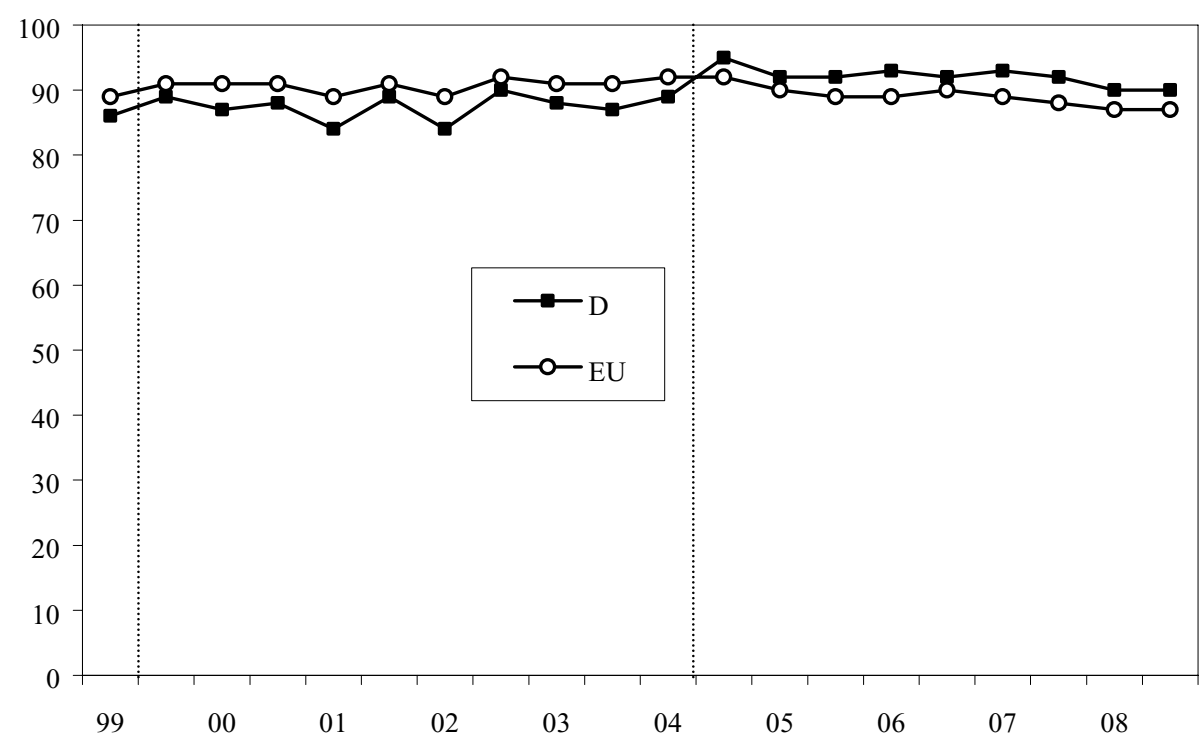

Quelle: Eurobarometerdaten.

Die Schaubilder 3 und 4 zeigen, dass das Europäische Parlament die bekannteste unter den fünf wichtigsten derjenigen europäischen Institutionen ist, deren Bekanntheitsgrad erfragt wurde. Von der Europäischen Kommission haben knapp vier Fünftel, vom Rat der Europäischen Union gut drei Fünftel der EU-Bürger etwas gehört, wobei sich der Bekanntheitsgrad im letzten Jahrzehnt nicht veränderte. Die Deutschen lagen lange Zeit unter dem europäischen Durchschnitt, in den letzten Jahren jedoch nicht mehr (Schaubild 3). 
Schaubild 3: Bekanntheitsgrad der Europäischen Kommission und des Rates der Europäischen Union 1999-2008 (Angaben in Prozent)

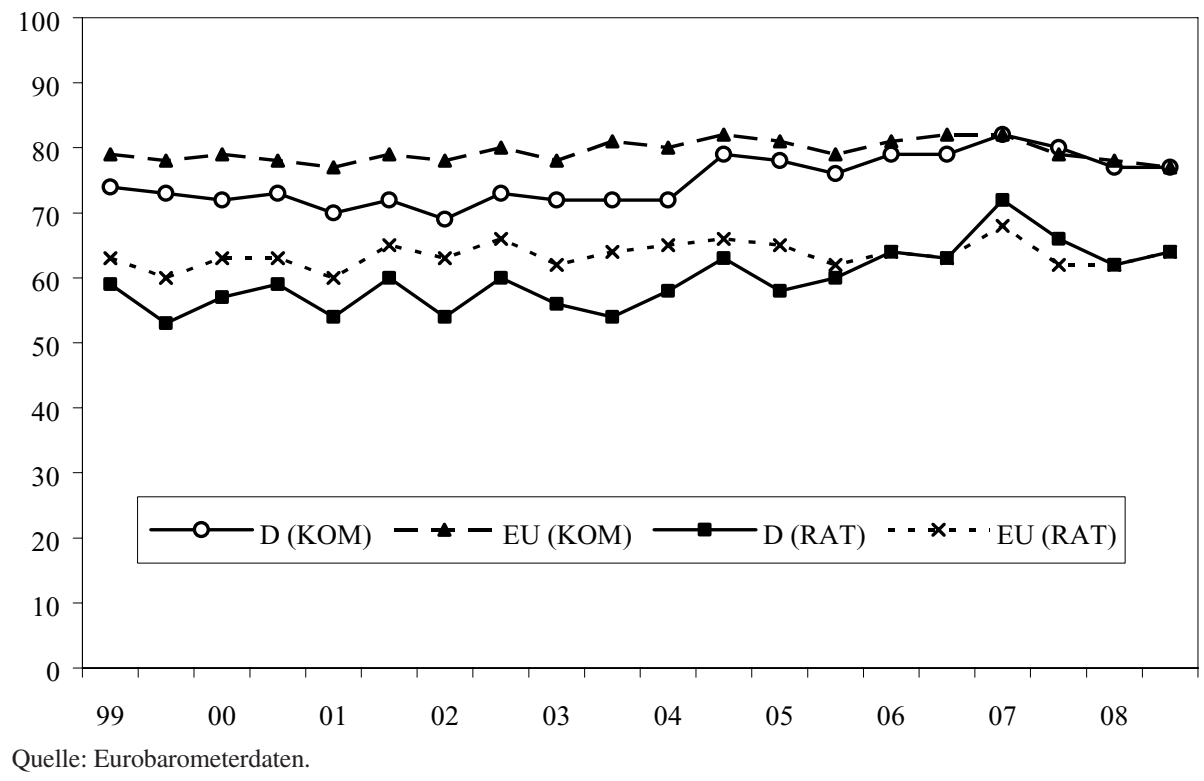

Schaubild 4: Bekanntheitsgrad des Europäischen Gerichtshofs und der Europäischen Zentralbank 1999-2008 (Angaben in Prozent)

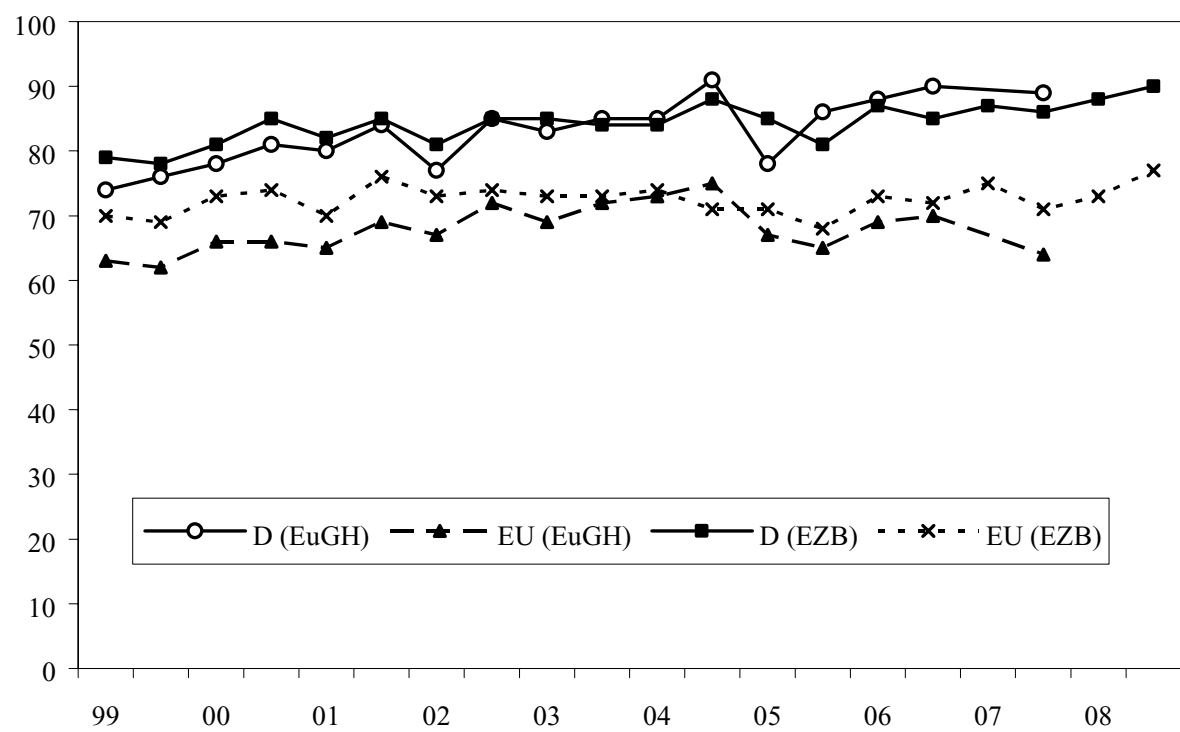

Quelle: Eurobarometerdaten. 
Überdurchschnittlich bekannt waren den Deutschen schon Ende der Neunzigerjahre der Europäische Gerichtshof (EuGH) und die Europäische Zentralbank (EZB) (siehe Schaubild 4). Während etwa zwei Drittel der Europäer schon einmal etwas vom EuGH und knapp drei Viertel schon einmal etwas von der EZB gehört oder gelesen haben - wobei die Bekanntheit der Europäischen Zentralbank im Zuge der beginnenden Medienberichterstattung über die internationale Finanzkrise im Herbst 2008 auf den bisher höchsten Wert gestiegen ist - kennen in jüngster Vergangenheit etwa neun von zehn Deutschen diese beiden Institutionen. Damit kommt ein besonderes Verhältnis der Deutschen zu einer bestimmten Art von Institutionen zum Ausdruck, auf das im nächsten Abschnitt näher eingegangen wird.

\section{Kenntnisse über das Europäische Parlament}

Wenn man in einer Befragung angibt, irgendwann schon einmal etwas über eine Institution gehört oder gelesen zu haben, impliziert das noch nicht, dass man auch - wenigstens rudimentäre - Kenntnisse über diese Institution besitzt. Zur umfassenden Analyse der kognitiven Orientierungen der Bevölkerung bezüglich des Europäischen Parlaments ist es daher notwendig, das Wissen der Bürgerinnen und Bürger über diese Institution zu untersuchen, wobei zu beachten ist, dass Wissensfragen in Umfragen nicht das objektive Wissen der Befragten, sondern subjektive Überzeugungen messen, die objektiv durchaus falsch sein können.

Leider existieren zu diesem Bereich keine über einen längeren Zeitraum hinweg vergleichbar erhobenen Daten. Im Frühjahr 1983, etwas mehr als ein Jahr vor den zweiten Direktwahlen, wurden die europäischen Bürger zu dessen Zusammensetzung und zum Rekrutierungsmodus befragt. Bei der Frage nach der Zusammensetzung waren drei Alternativen zur Gruppe der im Europäischen Parlament durch Vertreter repräsentierten Länder vorgegeben: „einige EG-Mitgliedsländer“, ,alle EG-Mitgliedsländer“ und ,,alle Länder Westeuropas“. Im Durchschnitt wählte nur gut die Hälfte der Befragten die richtige Alternative, bei den Deutschen waren es knapp zwei Drittel.

Die Frage nach dem Rekrutierungsmodus der Abgeordneten - mit den Alternativen ,,von der Regierung ernannt“ und ,,von den Bürgern gewählt“ - zeigte einen noch etwas geringeren Wissensstand. Hier wählten in mehr als der Hälfte der damaligen zehn EG-Länder weniger als die Hälfte der Befragten die richtige Alternative, nämlich die direkte Wahl der Abgeordneten durch die Bürger, bei den Deutschen war es gut die Hälfte. Bei beiden Fragen enthielt sich europaweit etwa ein Viertel der Befragten der Antwort.

Im Herbst 2002 wurde im Rahmen eines kurzen Wissenstests über die Europäische Union die Frage nach dem Rekrutierungsmodus erneut gestellt. ${ }^{5}$ Die Befragten sollten angeben, ob die Aussage, ,die Mitglieder des Europäischen Parlamentes werden von Bürgern wie uns gewählt", richtig oder falsch sei. Nur 38 Prozent der Deutschen und 43 Prozent im europäischen Durchschnitt gaben an, die Aussage sei richtig, 40 Prozent der Deutschen und 36 Prozent der Europäer insgesamt hielten sie für falsch, jeweils ein Fünftel konnte die Frage nicht beantworten (vergleiche Schaubild 5). Vom Frühjahr 2004 bis zum Frühjahr 2007 wurde erneut gefragt, ob die Aussage, ,die Mitglieder des Europäischen Parlamentes werden direkt von den Bürgern der Europäischen Union gewählt", nach Meinung der Befragten richtig oder falsch sei. Im Februar/März 2004, also drei Monate vor der fünften Direktwahl des Europäischen Parlaments, gaben 47 Prozent der Deutschen und 50 Prozent al-

5 Europäische Kommission: Eurobarometer. Die Öffentliche Meinung in der Europäischen Union, Bericht Nr. 58, März 2003, S. 15-16; Europäische Kommission: Eurobarometer. Public Opinion in the European Union, Report Number 58, März 2003, S. B.27. 
ler Europäer an, die Aussage sei richtig, nur noch 23 beziehungsweise 21 Prozent hielten sie für falsch. ${ }^{6}$ Vier bis fünf Monate nach der Direktwahl, im Oktober/November 2004, lag der Anteil der deutschen und gesamteuropäischen Bevölkerung, der auf diese Frage eine korrekte Antwort gab, bei 58 Prozent. ${ }^{7}$ Seither hat sich dieser Anteil wieder deutlich vermindert und ist im Frühjahr 2007 wieder in etwa auf den Stand vom Herbst 2002 gefallen. Gleichzeitig haben sich in Deutschland wie auch europaweit die Anteile derer erhöht, die die Aussage für falsch halten. Im Frühjahr 2007 hielten 42 Prozent der Deutschen die Aussage über die Direktwahl der Abgeordneten des Europäischen Parlaments für richtig, 44 Prozent waren hingegen der Überzeugung, sie sei falsch. ${ }^{8}$

\section{Schaubild 5: Kenntnis der Direktwahl des Europäischen Parlaments 2002-2007 (Angaben in Prozent)}

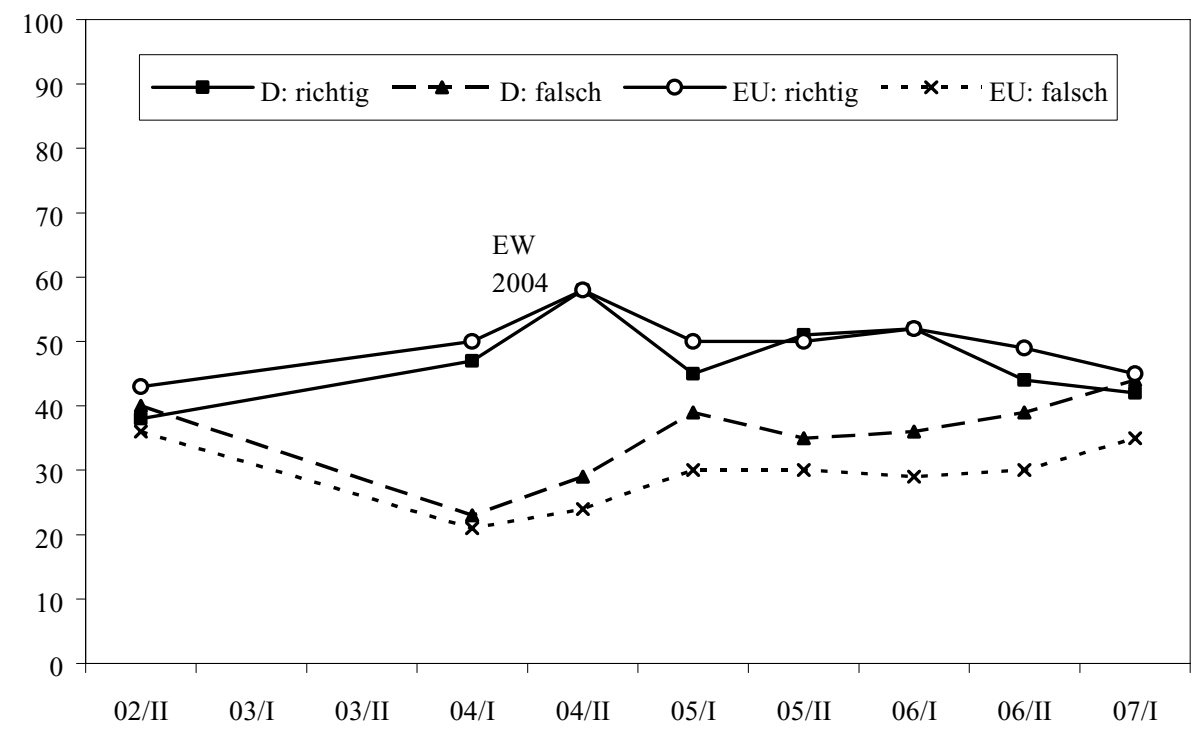

Quelle: Eurobarometerdaten.

Im Herbst 1988 und im Frühjahr 1989, also kurz vor den dritten Direktwahlen, wurden die Europäer zu den Kompetenzen des Europäischen Parlaments befragt. ${ }^{9}$ Es wurden ihnen drei als richtig oder falsch einzustufende Aussagen vorgelegt, die dem Europäischen Parla-

6 Europäische Kommission: Eurobarometer 61. Die öffentliche Meinung in der Europäischen Union. Frühjahr 2004, Juli 2004, S. B. 27; Europäische Kommission: Eurobarometer Spring 2004. Public Opinion in the European Union. Annexes, Mai 2004, S. T63-T64.

7 Gleichzeitig stimmten ein Drittel der Deutschen und ein knappes Drittel der Europäer insgesamt der Aussage zu, die letzten Wahlen zum Europäischen Parlament hätten im Juni 2002 stattgefunden, und nur 42 Prozent der Deutschen und 36 Prozent im europäischen Durchschnitt gaben korrekterweise an, diese Aussage sei falsch. Siehe Europäische Kommission: Eurobarometer 62. Die öffentliche Meinung in der Europäischen Union, Mai 2005, S. 45-46.

8 Europäische Kommission: Eurobarometer 67. Die Öffentliche Meinung in der Europäischen Union, November 2007, S. 128-131, Tabelle QA17.2.

9 Für eine ausführlichere europaweite Analyse dieser Daten vgl. Oskar Niedermayer: Europäisches Parlament und öffentliche Meinung, in: Oskar Niedermayer/Hermann Schmitt (Hrsg.): Wahlen und Europäische Einigung, Opladen 1994, S. $33 \mathrm{ff}$. 
ment die Macht zuschrieben, den Kommissionspräsidenten zu wählen, für jeden Mitgliedstaat bindende Gesetze zu beschließen und den EG-Haushalt abzulehnen. Auch diese Fragen enthüllten einen relativ geringen Informationsstand.

In Bezug auf die Wahl des Kommissionspräsidenten gab im europäischen Durchschnitt nur ein Sechstel, bei der Gesetzgebungskompetenz nur ein gutes Drittel der Bürger die korrekte Antwort, stufte die Aussagen also als falsch ein. In Deutschland war der Anteil der korrekten Antworten mit 29 und 52 Prozent deutlich höher. Auch die zunehmende Nähe zur Direktwahl hat das Wissen nicht vergrößert; im Gegenteil: Sowohl in Deutschland als auch in Gesamteuropa fiel der Prozentsatz der richtigen Antworten zwischen dem Herbst 1988 und dem Frühjahr 1989.

Ein überraschend hoher Wissensstand zeigte sich auf den ersten Blick bei der Frage nach der Kompetenz zur Haushaltsablehnung. Hier wurde europaweit von mehr als drei Fünfteln der Befragten die korrekte Antwort gegeben, also die Aussage als richtig eingestuft. Dies könnte durch die Medienberichterstattung über die Haushaltskonflikte zwischen dem Europäischen Parlament und dem Rat in der Vergangenheit verursacht sein. Die hohe Trefferquote könnte jedoch auch daraus resultiert haben, dass diejenigen, die ihr mehr oder minder differenziertes Image der (formalen) Machtfülle nationaler Parlamente einfach auf das Europäische Parlament übertrugen, ohne viel über das spezifische institutionelle System der Europäischen Union zu wissen, bei allen drei Aussagen die ,richtig'-Alternative wählten, was in Bezug auf das Europäische Parlament bei den ersten beiden Aussagen falsch, bei der dritten Aussage jedoch korrekt war. Einem solchen stereotypen EP-Bild als , machtvolles Parlament ' könnte auf der anderen Seite ein durch die Thematisierung der damaligen Kompetenzschwäche des Europäischen Parlaments in der massenmedialen Berichterstattung erzeugtes stereotypes Bild des Europäischen Parlaments als ,machtloses Parlament' gegenüberstehen.

Um der Hypothese eines bei einem beträchtlichen Teil der Deutschen und Europäer vorhandenen stereotypen EP-Bildes nachzugehen, wurden die drei Wissens-Fragen des Eurobarometers 31 vom Frühjahr 1989 näher analysiert. Einen ersten Hinweis auf die Gültigkeit der These eines stereotypen Bildes liefert die Beziehungsstruktur zwischen den drei Variablen. Ein stereotypes Bild als machtvolles beziehungsweise machtloses Parlament impliziert eine positive Beziehung zwischen allen drei Variablen, da das zugehörige individuelle Antwortmuster entweder die Bejahung oder die Verneinung jeweils aller drei Kompetenzen einschließt. Ein korrektes EP-Bild würde dagegen durch eine negative Beziehung der Variablen ,Kompetenz zur Haushaltsablehnung ' zu den anderen beiden Variablen angezeigt.

Die Analyse zeigte, dass die Beziehungen zwischen allen drei Variablen sowohl für die Gesamtheit der Europäer als auch für die Bürger jedes einzelnen Mitgliedslandes positiv waren. Allerdings ließ die Höhe der Koeffizienten den Schluss zu, dass stereotype EP-Bilder nicht vollständig dominieren. Dies wird auch deutlich, wenn man die kombinierten Antworten auf die drei Kompetenzfragen in fünf Kategorien aufteilt: (1) korrektes EP-Bild (alle drei Kompetenzfragen werden richtig beantwortet), (2) machtvolles Parlament (dem Europäischen Parlament werden alle drei Kompetenzen zugeschrieben), (3) machtloses Parlament (dem Europäischen Parlament werden gar keine Kompetenzen zugeschrieben), (4) sonstige Einschätzung (alle anderen möglichen Antwortkombinationen) und (5) weiß nicht/keine Antwort (mindestens zu einer der Kompetenzfragen keine klare Meinung).

Die Typologie verdeutlichte, dass wenige Monate vor der dritten Direktwahl nur sehr wenige europäische Bürger eine korrekte Vorstellung darüber hatten, welche Kompetenzen das Europäische Parlament besaß. Im europäischen Durchschnitt waren es 4 Prozent, bei den Deutschen 7 Prozent. Weitaus am stärksten verbreitet war hingegen das stereotype EP-Bild des , machtvollen Parlaments'. Deutlich mehr als ein Drittel der Deutschen und der Europäer 
insgesamt schrieb dem Europäischen Parlament Kompetenzen in allen drei Bereichen zu. Ein stereotypes EP-Bild des, machtlosen Parlaments' war dagegen wenig zu finden. Nur 5 Prozent der Europäer und 9 Prozent der Deutschen perzipierte das Europäische Parlament auf diese Weise. Gleichgültigkeit, Desinteresse oder zumindest Unsicherheit dokumentierte europaweit etwa ein Fünftel.

Eine Validierung der für 1989 gefundenen Ergebnisse mit der Möglichkeit, ohne neuere Daten zur Perzeption der EP-Kompetenzen ${ }^{10}$ abzuschätzen, ob stereotype Bilder des Europäischen Parlaments auch heute noch existieren, ergibt sich, wenn man eine weitere Frage heranzieht, die in den Umfragen regelmäßig gestellt wird: die generelle Einschätzung der Wichtigkeit der Rolle des Europäischen Parlaments im Leben der Europäischen Union. ${ }^{11}$ Wird diese Frage von den Bürgern gemäß ihres generellen EP-Bildes beantwortet, so müsste sich eine positive Beziehung zwischen den beiden Variablen dergestalt zeigen, dass mit zunehmend ,machtvollem 'EP-Bild auch die Rolle des Europäischen Parlaments im Rahmen der Europäischen Union als immer wichtiger eingestuft wird. In der Tat zeigte sich eine solche Beziehung: Die Gruppe der Befragten mit einem korrekten EP-Bild war in ihrer Einschätzung der EP-Rolle als eher wichtig oder eher unwichtig gespalten, die Gruppe mit dem ,machtlosen 'EP-Bild schätzte die Rolle überwiegend als eher unwichtig ein und diejenigen, die das Europäische Parlament von seiner Kompetenzausstattung her als machtvoll perzipierten, gingen zu drei Vierteln auch von einer eher wichtigen Rolle des Parlaments im Leben der Europäischen Union aus.

Die Schaubilder 6 und 7 geben die von der Bevölkerung perzipierte Wichtigkeit der Rolle des Europäischen Parlaments im Institutionensystem der Europäischen Union von 1977 bis 1998 und von 1999 bis 2008 wieder. ${ }^{12}$ Ab 1999 ist es zudem möglich, die Einschätzung des Europäischen Parlaments mit der Perzeption der Wichtigkeit der anderen relevanten EU-Institutionen zu vergleichen (siehe die Schaubilder 8 und 9). Ein solcher Vergleich zeigt, dass das Europäische Parlament sowohl von den Deutschen als auch von den Europäern insgesamt im Vergleich der drei primär die Politik- und Systemgestaltungsfunktion wahrnehmenden Institutionen als die wichtigste angesehen wird. Die Kommission liegt an zweiter Stelle und der Rat wird als relativ am wenigsten wichtig angesehen, wobei die Relevanzeinschätzung aller drei Institutionen in Deutschland im Zeitablauf noch etwas zugenommen hat und jetzt im europäischen Durchschnitt liegt.

Der Europäische Gerichtshof und die Europäische Zentralbank sind den Deutschen schon seit Ende der Neunzigerjahre nicht nur überdurchschnittlich bekannt (vergleiche Schaubild 4), sie werden auch als überdurchschnittlich wichtig eingeschätzt und reichen fast schon an das Europäische Parlament heran beziehungsweise übertreffen es sogar (EZB).

10 Leider wurden die Kompetenzfragen nicht wieder gestellt.

11 Der Fragetext lautete von 1977 bis 1998: „Wie wichtig ist Ihrer Meinung nach die Rolle, die das Europa-Parlament heute im Leben der Europäischen Gemeinschaft spielt? Ist sie sehr wichtig, wichtig, nicht sehr wichtig oder überhaupt nicht wichtig?".

12 Im Jahre 1999 wurden sowohl die Frageformulierung als auch die Antwortkategorien geändert. Gefragt wurde nun: „Sagen Sie mir bitte für jede dieser europäischen Einrichtungen, ob sie Ihrer Meinung nach eine wichtige Rolle im Leben der Europäischen Union spielt oder nicht?“" Als Antwortmöglichkeiten sind jetzt nur noch „wichtige Rolle“ und „keine wichtige Rolle“ vorgesehen. 
Schaubild 6: Perzipierte Wichtigkeit der Rolle des Europäischen Parlaments im Institutionensystem der EU 1977-1998 (Mittelwerte von 1 = überhaupt nicht wichtig bis 4 = sehr wichtig)

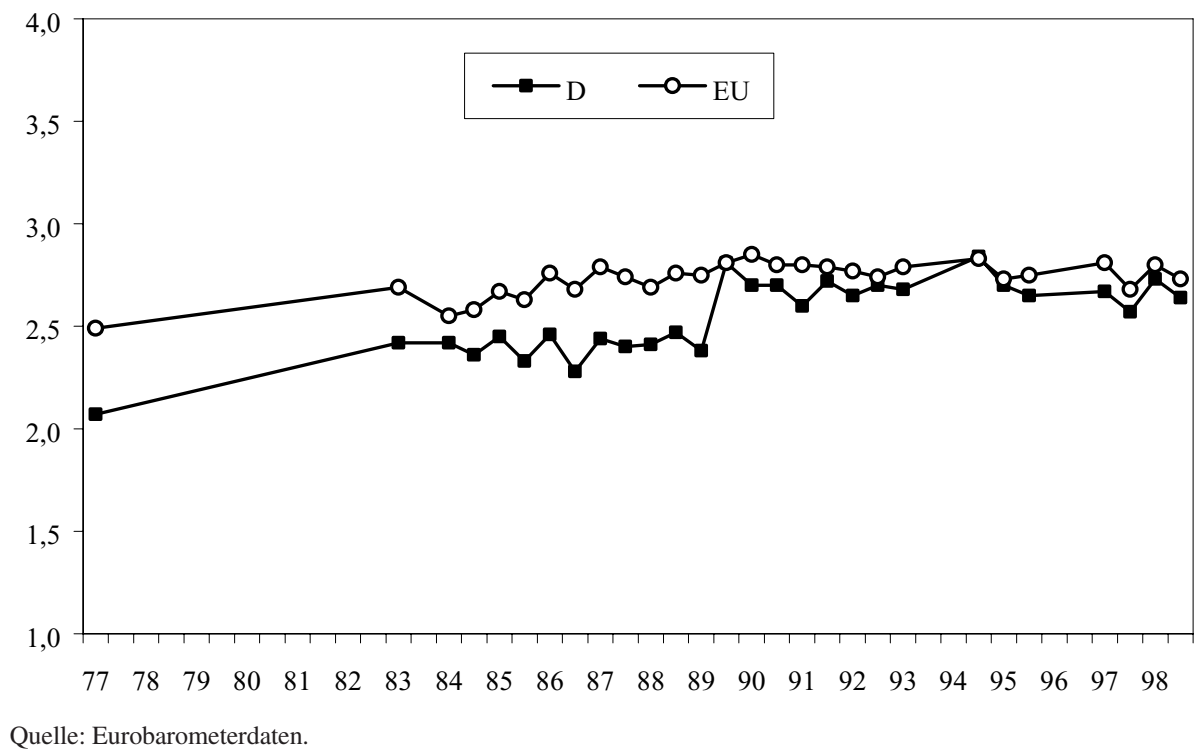

Schaubild 7: Perzipierte Wichtigkeit der Rolle des Europäischen Parlaments im Institutionensystem der EU 1999-2008 (Angaben in Prozent, wichtige Rolle“)

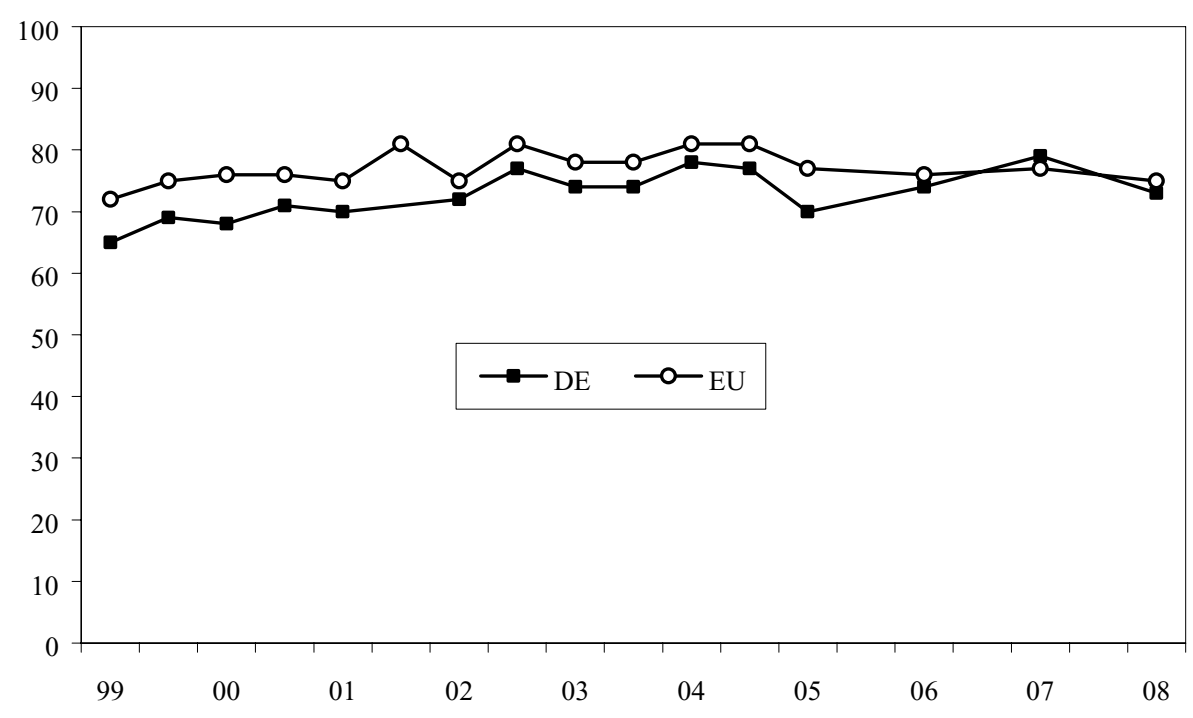

Quelle: Eurobarometerdaten. 
Schaubild 8: Perzipierte Wichtigkeit der Rolle der Europäischen Kommission und des Rats im Institutionensystem der EU 1999-2008 (Angaben in Prozent)

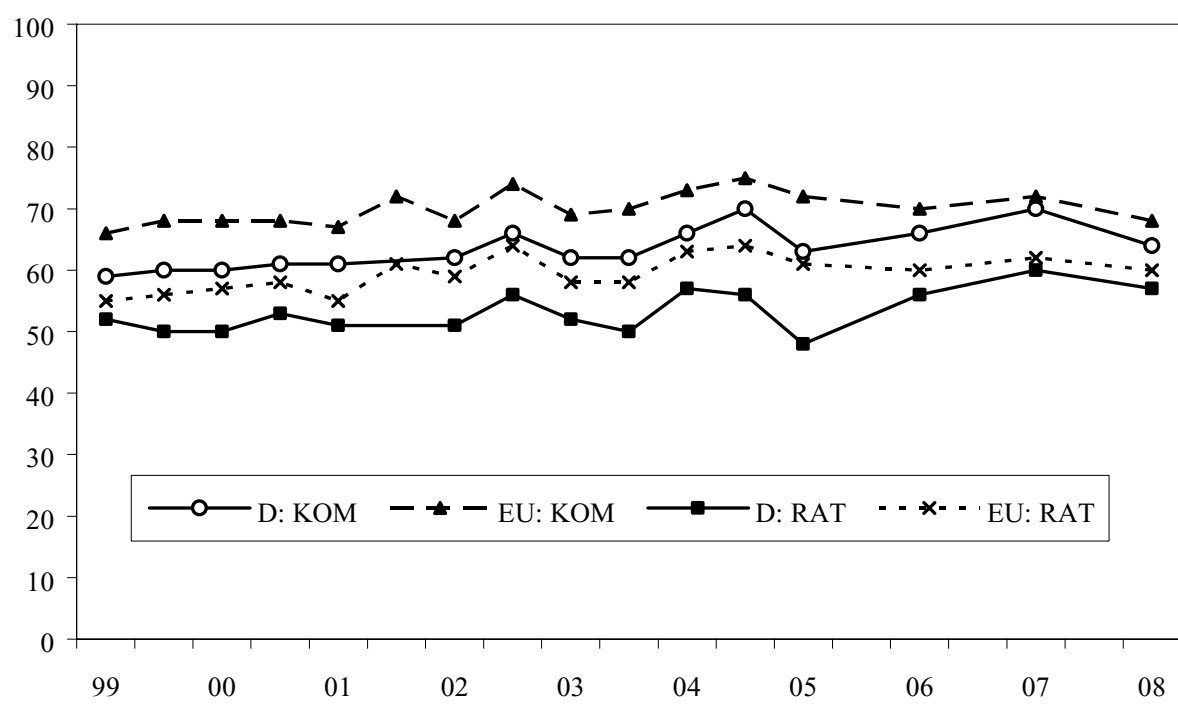

Quelle: Eurobarometerdaten.

Schaubild 9: Perzipierte Wichtigkeit der Rolle des Europäischen Gerichtshofs und der Europäischen Zentralbank im Institutionensystem der EU 1999-2008 (Angaben in Prozent)

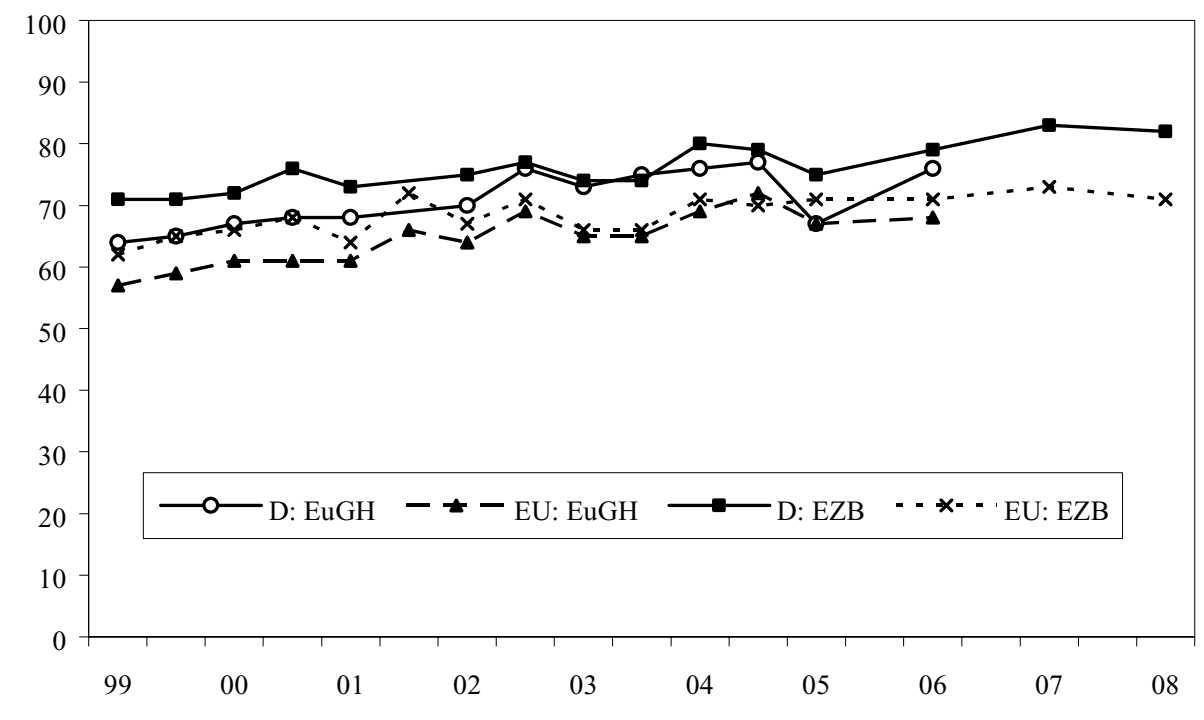

Quelle: Eurobarometerdaten. 
Betrachtet man die Perzeption der Wichtigkeit der Rolle des Europäischen Parlaments durch die Deutschen und die Europäer in den letzten drei Jahrzehnten, so zeigt sich keine systematische Beziehung zwischen der Wichtigkeitsperzeption des Parlaments und der in dieser Zeit erfolgten sukzessiven Erweiterung seiner Kompetenzen.

Die erste Direktwahl des Europäischen Parlaments im Jahre 1979 vergrößerte zwar seine demokratische Legitimation und steigerte sein Selbstbewusstsein, war aber nicht mit einer Ausweitung seiner Kompetenzen verbunden. Dennoch wurde seine Rolle im Institutionensystem der Europäischen Union nach der Wahl sowohl in Deutschland als auch im europäischen Durchschnitt von der Bevölkerung als deutlich wichtiger angesehen (vergleiche Schaubild 6). Die sukzessiven Erweiterungen der Kompetenzen des Europäischen Parlaments durch die Einheitliche Europäische Akte (unterzeichnet 1986, in Kraft seit 1987), den Vertrag von Maastricht (unterzeichnet 1992, in Kraft seit 1993), den Vertrag von Amsterdam (beschlossen 1997, in Kraft seit 1999) und den Vertrag von Nizza (unterzeichnet 2001, in Kraft seit 2003) haben dagegen weder bei den Deutschen noch im europäischen Durchschnitt zu jeweiligen Steigerungen der Wichtigkeitsperzeption des Europäischen Parlaments im Institutionensystem der Europäischen Union geführt. Das heißt: Die im Vorfeld der jeweiligen Vertragsunterzeichnung und während der Ratifizierungsprozesse geführten Diskussionen um die Stärkung der Rolle des Europäischen Parlaments und die mit den Verträgen tatsächlich erfolgten Kompetenzerweiterungen sind in den Bevölkerungen nicht in einer Weise wahrgenommen worden, die zu systematischen Veränderungen der kognitiven Orientierungen geführt hätte. ${ }^{13}$

Auch dies lässt vermuten, dass bei vielen Bürgerinnen und Bürgern bis heute stereotype EP-Bilder existieren - und die Antwortverteilungen im Vergleich mit der Einschätzung der beiden anderen im engeren Sinne ,politischen', also primär die Politik- und Systemgestaltungsfunktion wahrnehmenden Institutionen sprechen für eine Dominanz des Bildes vom , machtvollen Parlament', das im Institutionensystem der Europäischen Union eine führende Rolle spielt.

\section{Evaluative Orientierungen gegenüber dem Europäischen Parlament}

Hierzu passt auch, dass bei den affektiv-wertbezogenen Beurteilungen dieser drei Institutionen durch die Bürgerinnen und Bürger in Form des in sie gesetzten Vertrauens das Europäische Parlament sowohl in Deutschland als auch im gesamteuropäischen Durchschnitt am besten abschneidet. Die Schaubilder 10 und 11 verdeutlichen dies von 1999 bis 2008 am Index des sogenannten ,Netto-Vertrauens", der statt des Prozentsatzes derjenigen, die der jeweiligen Institution vertrauen, die Differenz zwischen den Prozentsätzen der positiven und der negativen Antworten angibt, also das Ausmaß aufzeigt, in dem das Vertrauen das Misstrauen überwiegt. Die Verwendung des Indexes erfolgt aufgrund der Überlegung, dass es bei der Interpretation der Ergebnisse einen wesentlichen Unterschied macht, ob einem bestimmten Ausmaß an Vertrauen ein hohes, möglicherweise das Vertrauen sogar übertreffendes Ausmaß an Misstrauen gegenübersteht, oder ob der Rest der Bevölkerung eher indifferent ist.

13 Und dies obwohl z.B. in Deutschland die Ratifizierung des Maastrichter Vertrags von einer Verfassungsklage gegen die parlamentarische Zustimmung begleitet wurde. 
Schaubild 10: Netto-Vertrauen in das Europäische Parlament, die Kommission und den Rat: Deutschland 1999-2008 (Angaben in Prozent)

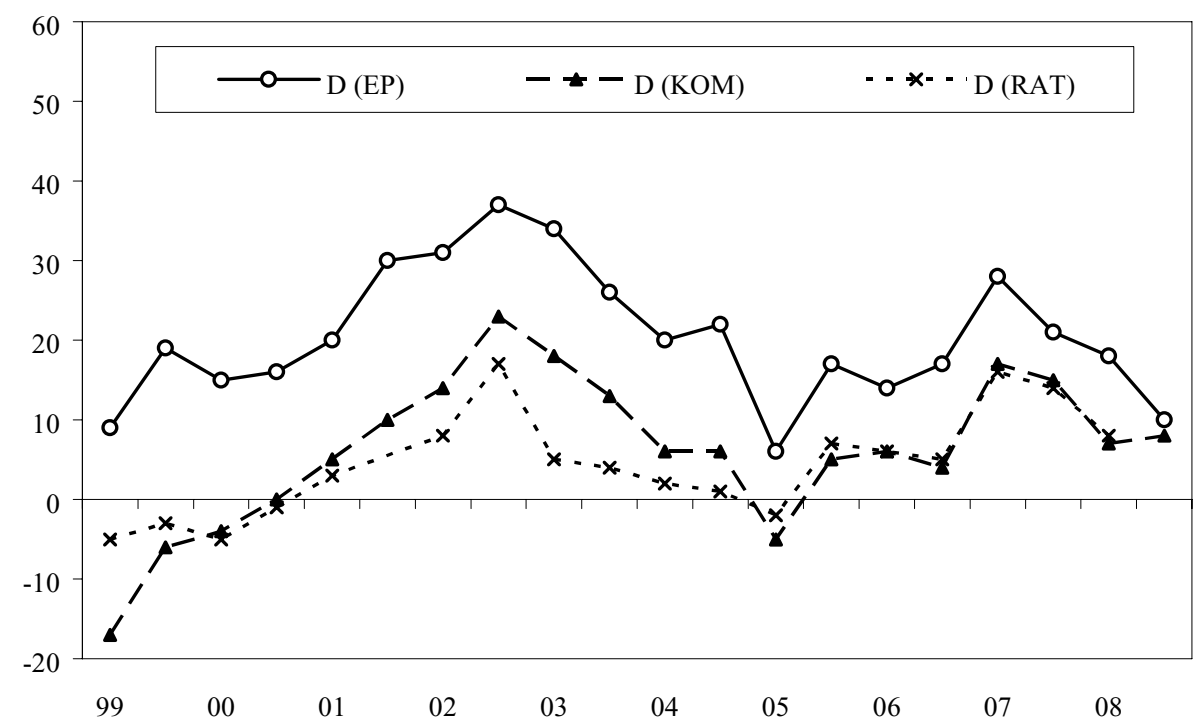

Quelle: Eurobarometerdaten.

Schaubild 11: Netto-Vertrauen in das Europäische Parlament, die Kommission und den Rat: europäischer Durchschnitt 1999-2008 (Angaben in Prozent)

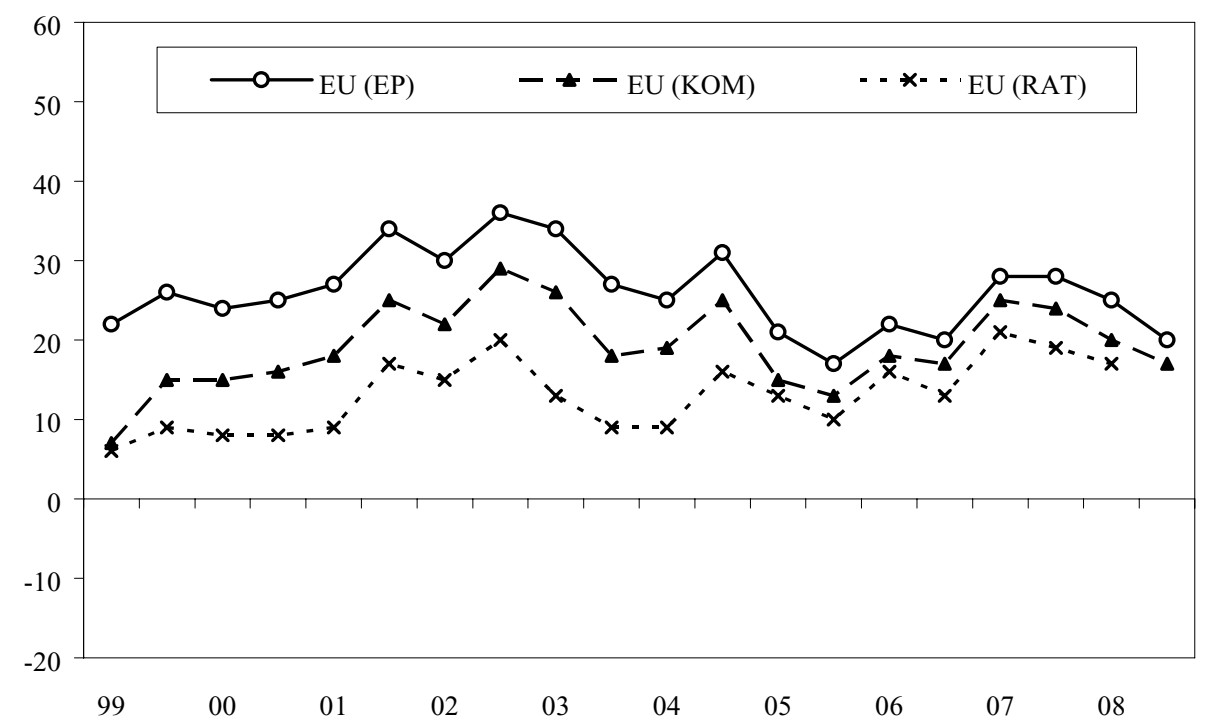

Quelle: Eurobarometerdaten. 
Die beiden Schaubilder machen auch deutlich, dass das Vertrauen in alle drei Institutionen in Deutschland fast immer mehr oder minder deutlich unter dem europäischen Durchschnitt liegt, die Deutschen somit den im engeren Sinne, politischen 'EU-Institutionen etwas kritischer gegenüberstehen als die Europäer insgesamt. Dies zeigt sich vor allem bei der Kommission und dem Rat: Während im europäischen Durchschnitt über das gesamte letzte Jahrzehnt hinweg bei beiden Institutionen der Index des Netto-Vertrauens immer positiv war, das Vertrauen das Misstrauen also immer überwog, war dies in Deutschland vor allem Ende der Neunzigerjahre nicht der Fall. Am stärksten zeigte sich dies an den Werten für die Europäische Kommission im Frühjahr 1999, als gegen die damalige Kommission unter dem Präsidenten Jacques Santer schwerwiegende Anschuldigungen erhoben wurden (Betrug, Missmanagement und Nepotismus), die zu - abgelehnten - Misstrauensanträgen gegen die Kommission im Europäischen Parlament, zur Einsetzung eines Untersuchungsausschusses unabhängiger Sachverständiger und letztlich im März 1999 zum geschlossenen Rücktritt aller Kommissionsmitglieder führten. ${ }^{14}$

Das Schaubild 12 zeigt die Entwicklung des Vertrauens in Deutschland und der Europäischen Union insgesamt gegenüber dem Europäischen Gerichtshof und der Europäischen Zentralbank.

\section{Schaubild 12: Netto-Vertrauen in den Europäischen Gerichtshof und die Europäische Zentralbank 1999-2008 (Angaben in Prozent)}

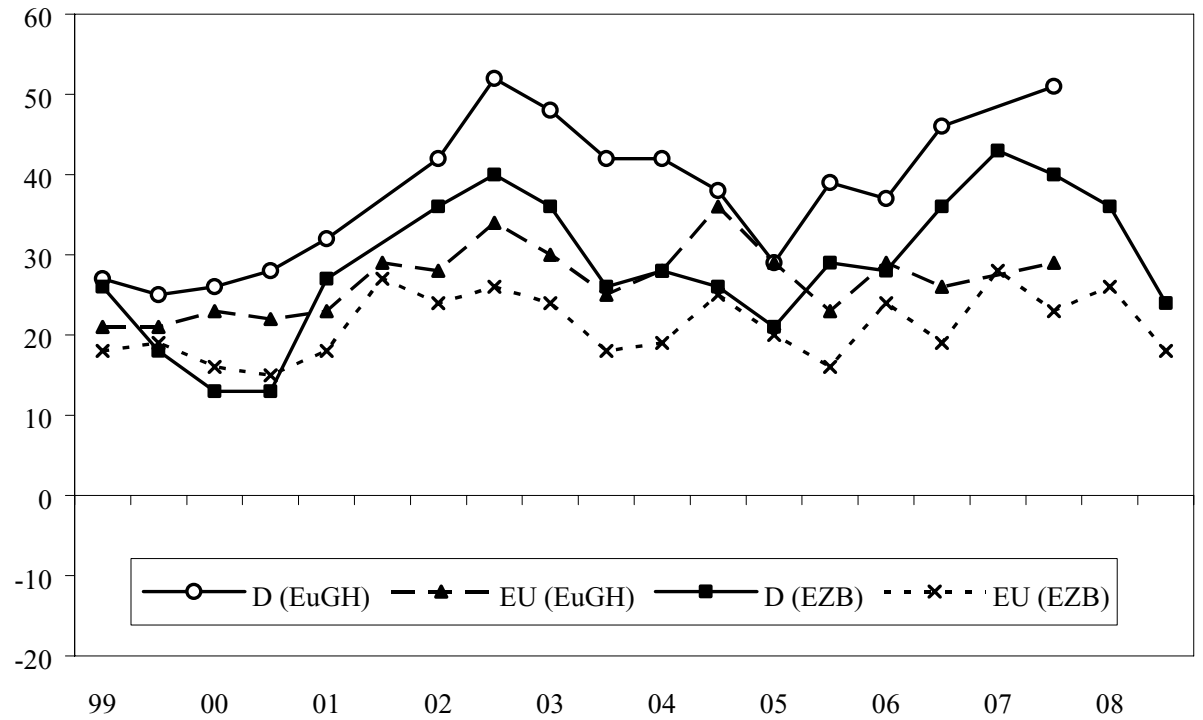

Quelle: Eurobarometerdaten.

Während das Vertrauensniveau bezüglich des Europäischen Parlaments, der Kommission und des Rates in Deutschland in der Regel unterdurchschnittlich ist, liegt es bezüglich dieser

14 Während im Frühjahr 1999 im gesamteuropäischen Durchschnitt 40 Prozent der Befragten der Kommission noch eher vertrauten und 33 Prozent ihr eher nicht vertrauten, vertrauten ihr in Deutschland nur 28 Prozent und 45 Prozent taten es nicht. 
beiden Institutionen fast die gesamte Zeit mehr oder weniger deutlich über dem gesamteuropäischen Durchschnitt.

Vergleicht man das Ausmaß, in dem die Deutschen den fünf hier betrachteten Institutionen im letzten Jahrzehnt Vertrauen entgegengebracht haben, so genoss stets der Europäische Gerichtshof das größte Vertrauen, gefolgt von der Europäischen Zentralbank. Erst danach kamen das Europäische Parlament, die Europäische Kommission und der Rat der Europäischen Union. Die Deutschen schenken somit ihr Vertrauen am stärksten denjenigen Institutionen, die mit der Sicherstellung der Einhaltung allgemeiner Regelungen befasst und kontroversen inhaltlich-politischen Diskussionen eher entzogen sind.

Dies entspricht durchaus auch der Struktur des Vertrauens der Deutschen in ihre nationalen politischen Institutionen. ${ }^{15}$ Im nationalen Kontext wird den rechtsstaatlichen Institutionen (Bundesverfassungsgericht, Gerichtsbarkeit allgemein, Polizei) ein deutlich größeres Vertrauen entgegengebracht als den parteienstaatlichen Institutionen (Bundestag, Bundesregierung), die in die kontroversen inhaltlich-politischen Diskussionen einbezogen sind.

\section{Fazit}

Die Analyse hat gezeigt, dass das Europäische Parlament bei den Deutschen wie auch bei den Europäern insgesamt die bekannteste europäische Institution ist. Im Vergleich mit der Europäischen Kommission und dem Rat der Europäischen Union wurde zudem deutlich, dass das Europäische Parlament von den drei primär die Politik- und Systemgestaltungsfunktion wahrnehmenden europäischen Institutionen das größte Vertrauen genießt und als die wichtigste angesehen wird.

Auch wenn diese Orientierungen gegenüber dem Europäischen Parlament bei einem beträchtlichen Teil der Bevölkerung wohl eher auf stereotypen Parlamentsbildern als auf einem soliden Wissensfundament über diese Institution beruhen, wie anhand verschiedener Indikatoren deutlich wurde, so bedeutet dies doch, dass im subjektiven Überzeugungssystem eines Großteils der Bürgerinnen und Bürger das Europäische Parlament keine machtlose, Quasselbude', sondern ein zentraler Akteur des Institutionensystems der Europäischen Union ist.

Warum sind dann bei den Direktwahlen zu dieser Institution so wenig Bürgerinnen und Bürger daran interessiert, von ihrem demokratischen Mitgestaltungsrecht Gebrauch zu machen ${ }^{16}$ Das wesentliche Argument zur Auflösung dieses scheinbaren Widerspruchs besteht in der Tatsache, dass die untersuchten Orientierungen sich auf die Sichtbarkeit, Rolle und Bewertung des Europäischen Parlaments auf der europäischen Ebene beziehen. Wie sich anhand einer Fülle von Belegen zeigen lässt, messen die Bürgerinnen und Bürger jedoch dieser Politikebene im Allgemeinen und damit auch dem Europäischen Parlament im Besonderen noch immer nicht annähernd die Relevanz zu, die realiter gegeben ist.

So zeigten beispielsweise die Eurobarometerumfragen der Jahre 2002 bis 2004, dass nur etwa ein Siebtel der Deutschen und auch der übrigen Europäer der Auffassung waren, dass die Tätigkeiten und Entscheidungen des Europäischen Parlaments große Auswirkungen auf die Bürger haben würden. Etwa ein Fünftel der Befragten war der Auffassung, die Arbeit des Europäischen Parlaments hätte überhaupt keinen Einfluss auf die Bürger. Für die Bundesrepublik zeigen zudem die jeweiligen Vorwahlumfragen zu allen bisherigen Direktwahlen von 1979 bis heute, dass die Deutschen eine klare Rangfolge internalisiert haben, wenn sie nach der Relevanz der Entscheidungen der Parlamente auf den verschiedenen Ebenen für

15 Vgl. hierzu Oskar Niedermayer: Bürger und Politik. Politische Orientierungen und Verhaltensweisen der Deutschen, 2. Auflage, Wiesbaden 2005, S. 64-86.

16 Bei der Europawahl vom 7. Juni 2009 betrug die Wahlbeteiligung nur 43 Prozent. 
sie persönlich gefragt werden: Dem Bundestag wird die größte Relevanz zugemessen, an zweiter Stelle kommen die Landtage, dann folgen die Gemeinderäte und an letzter Stelle steht immer das Europäische Parlament.

Solange die lebensweltliche Alltagsrelevanz der Entscheidungen des Europäischen Parlaments noch nicht genügend in den Köpfen der Bürgerinnen und Bürger verankert ist, solange kann auch nicht von einer ausreichenden Erfüllung der Interaktionsfunktion des Europäischen Parlaments gesprochen werden. Schuld an dieser Situation, das muss betont werden, trägt jedoch bei Weitem nicht alleine das Europäische Parlament.

\section{Osteuropa}

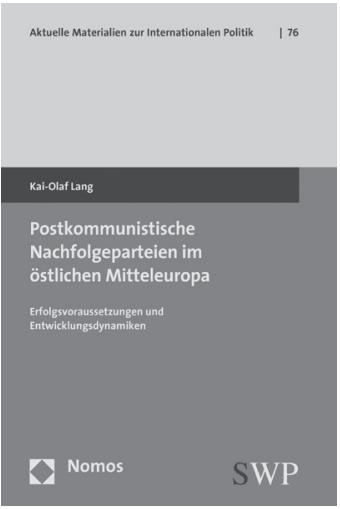

\section{Postkommunistische Nachfolgeparteien} im östlichen Mitteleuropa

Erfolgsvoraussetzungen und Entwicklungsdynamiken

Von Dr. Kai-Olaf Lang 2009, 359 S., brosch., 59,- $€$, ISBN 978-3-8329-3642-6

(Aktuelle Materialien zur Internationalen Politik, $B d .76)$

\section{Die Integration Südosteuropas} Die Demokratisierungspolitik europäischer Organisationen in Albanien Von Dr. Judith Hoffmann 2008, 380 S., brosch., 59,- $€$, ISBN 978-3-8329-4075-1

Bitte bestellen Sie im Buchhandel oder versandkostenfrei unter $\downarrow$ www.nomos-shop.de

\section{Fördermittel statt} Beitrittsperspektive Brüssel und Chisinau seit der Auflösung der Sowjetunion Von Dr. Victoria Reinhardt 2008, 268 S., brosch., 39,- $€$, ISBN 978-3-8329-3815-4 (Nomos Universitätsschriften - Politik, $B d .162$ )

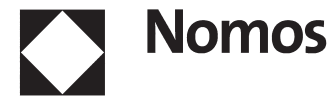

